\title{
Crop Disease Prediction using Deep Convolution Neural Network
}

\author{
N.Dhanalakshmi ${ }^{1}$,M.Jeyanthi², A V Prabu ${ }^{3}$ \\ ${ }^{1}$ Professor,Department of Computer Science and Engineering, PSNA College of Engineering and Technology,Dindigul \\ ${ }^{2}$ Assistant Professor,Department of Computer Science and Engineering PSNA College of Engineering and Technology,Dindigul. \\ ${ }^{3}$.Dept of ECE, Associate Professor,Koneru Lakshmaiah Education Foundation, Vaddeswaram, Guntur, Andhra Pradesh, India.
}

\begin{abstract}
Harvest ailment has been a significant danger to ranchers thereby decreasing the harvest yield and bargaining its quality. One of the huge difficulties is a precise determination of illnesses. Generally, recognizable proof of yield infections has depended on human explanation by visual review. These days different current advancements have developed to limit post reap preparing, to strengthen horticultural manageability and to boost the profitability. In this work utilizes Deep Convolution Neural Network in distinguishing among sound and unhealthy leaf from the informational collections made. Our proposed paper incorporates different periods of execution specifically dataset obtaining, include extraction, preparing the classifier and grouping. The gathered datasets of both unhealthy and solid leaves are prepared under CNN to arrange the sick and sound pictures. For removing highlights of a picture by utilizing GLCM. So as to assess the exhibition of CNN model is done through confusion matrix which shows $82.39 \%$ the exactness of the model by utilizing TP and TN tests.
\end{abstract}

Keywords

CNN,GLCM,TP,TN

Article Received: 20 September 2020, Revised: 30 November 2020, Accepted: 18 December 2020

\section{Introduction}

Plant diseaseshas for quite some time been one of the significant dangers to food security. Distinguishing indications and realizing how to adequately control sicknesses in plant is a continuous test. An underlying endeavor to utilize profound learning for picture based plant illness determination was accounted for in 2016, where the prepared model had the option to characterize 14 yields and 26 sicknesses with a precision of $99.35 \%$ against optical pictures [6]. From that point forward, progressive ages of profound learning-based sickness conclusion in different yields have been accounted for [1 - 7]. Governments use crop type planning for avocation of sponsorships given to ranchers, misrepresentation location and protection claims. Notwithstanding every one of these advantages, analysts are keen on phonological measurements got through plant checking because of their expected an incentive as immediate pointers of environmental change.

Significant Convolutional Neural Network (CNN) is a remarkable sort of Neural Networks, which has shown commendable execution on a couple of contentions related to Computer Vision and Image Processing. A part of the invigorating application zones of $\mathrm{CNN}$ fuse Image Classification and Segmentation, Object Detection, Video Processing, Natural Language Processing, and Speech Recognition. The unbelievable learning limit of significant $\mathrm{CNN}$ is generally a result of the use of various component extraction masterminds that can therefore take in depictions from the data. Convolution neural associations are neural associations used on a very basic level to arrange pictures bunch pictures by its equivalence and to do fight affirmation. CNN picture portrayals takes a data picture which is as show of pixels and the assessment of cycle it and orchestrate it under explicit orders as bunch of pixels and it depends upon the image objective. As a matter of fact,

significant learning CNN models to get ready and test, every data picture will go it through a movement of convolution layers with channels (Kernals), Pooling, totally related layers (FC).Every $\mathrm{CNN}$ is included different layers, the three essential sorts of layers are convolutional, pooling, and totally related, as Fig.1 CNN Model . All of these layers is made of centers that see some data and produce a yield.

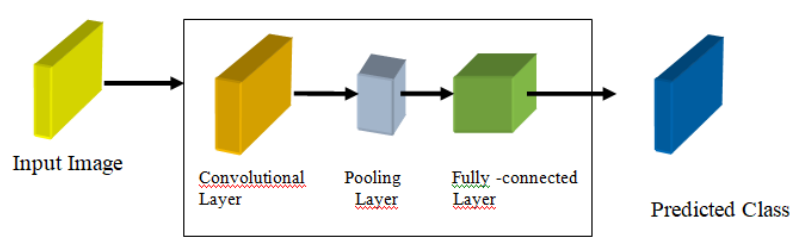

Fig.1 CNN Model

\section{Related Work}

[8]In late occasions, worker based and portable based methodology for ailment distinguishing proof has been utilized for ailment ID. A few elements of these advancements being high goal camera, elite handling and broad inherent adornments are the additional preferences bringing about programmed disease acknowledgment. Current methodologies, for example, AI and profound learning calculation have been utilized to expand the acknowledgment rate and the exactness of the outcomes. Different explores have occurred under the field of AI for plant infection discovery and finding, such customary AI approach being arbitrary backwoods, fake neural organization, uphold vector machine(SVM), fluffy rationale, 
K-implies technique, Convolution neural organizations and so forth....

Irregular timberlands are overall, learning technique for arrangement, relapse and different assignments that work by developing a backwoods of the choice trees during the preparation time. Dissimilar to choice trees, Random woodland beat the inconvenience of over fitting of their preparation informational index and it handles both numeric and downright information. The CNN system is a significant model for profound learning hypothesis, with a wide scope of utilizations in picture acknowledgment and arrangement $[9,10]$. It is created from fake neural organizations. The past layer is utilized as the contribution of the ensuing layer, and the back-spread calculation is utilized to refresh the boundaries.

\section{Methodology}

3.

\subsection{Data acquisition}

The PlantVillage image dataset collected form github.com and out of this ,80\% of knowledge used for training and remaining $20 \%$ used as a test dataset. This dataset contains 38 categories of diseased or healthy leaf images.In this step the sample images are collected, which are required tocoach thesystem.

\subsection{Building the convolution neural network}

The CNN reformed this field by learning the fundamental shapes inside the primary layers and advancing to discover highlights of the picture inside the more profound layers, prompting more precise picture order. Convolution neural organizations, or CNNs, are profound learning model which has at least an info layer, one concealed layer and a yield layer. Principle highlights of $\mathrm{CNN}$ are abuse of the convolution activity, post-convolution pooling, non-straight actuation capacities and move learning and profound component extraction.

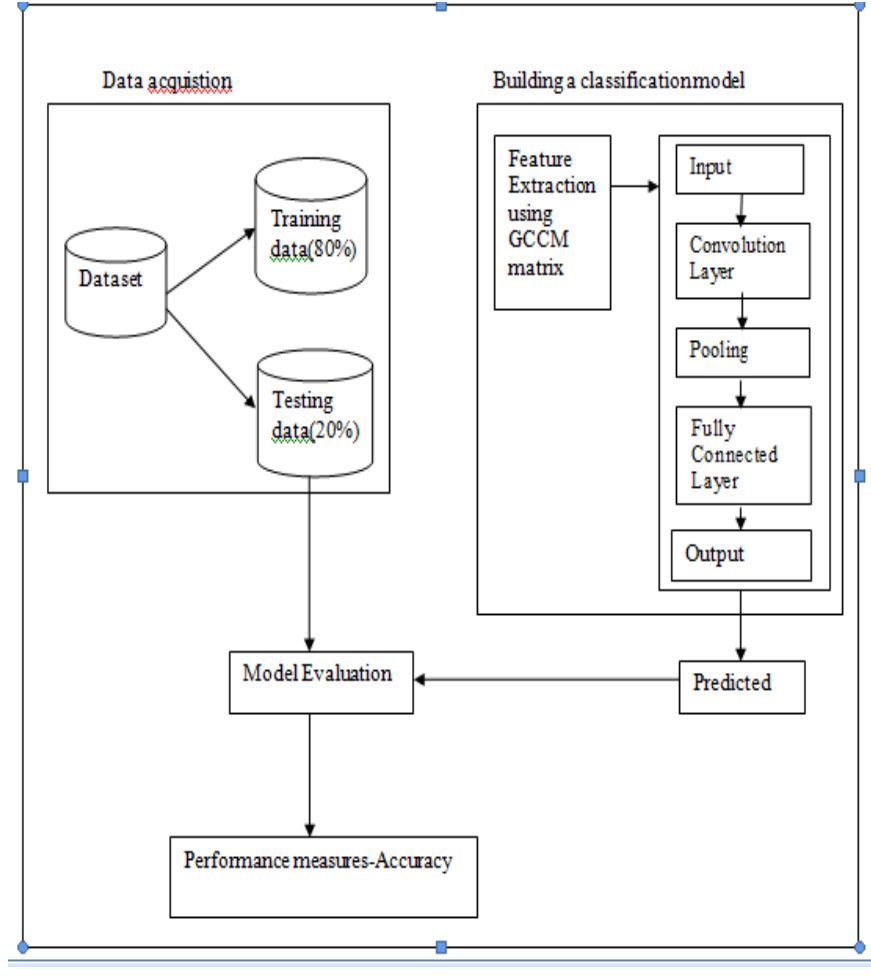

Fig 2. System Architecture

\subsubsection{Featureextraction}

The feature extraction is employed to extract the knowledge which will be wont to determine the importance of the given sample. the most sorts of features are shape, color and texture, which are mostly utilized in image processing technique.color and texture features both are extracted to urge better accuracy.Surface might be a component wont to segment pictures into areas of intrigue and to order those locales which gives data inside the separating of tones or powers in an image. Texture is described by the spatial dispersion of force levels during an area.

Simple Analysis of Texture
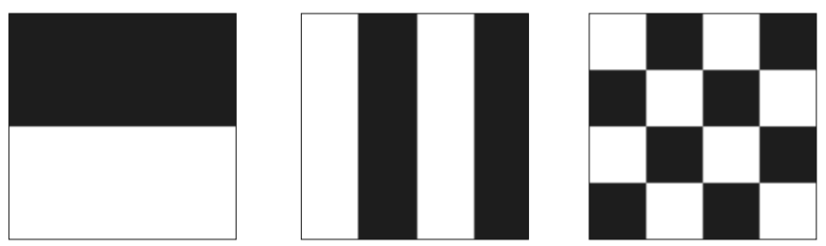

For instance, an image includes a half dark and half white dispersion of pixels. Three unique pictures with an equal force circulation, yet with various surfaces. Surface is also a blend of rehashed designs with a day by day recurrence. Surface examination is characterized in light of the fact that the grouping or division of textural highlights concerning the type of a little component, thickness and heading of normality. for the most part, the applications include the robotized extraction of highlights from the picture which is then utilized for a spread of division and order undertakings, such as recognizing ordinary tissue from unusual tissue. 
Contingent on the real 70 characterization task, the separated highlights catch morphological properties, shading properties, or certain textural properties of the picture. Highlights regularly contain data comparative with dim shade, surface, shape, or setting. To characterize an item in an image, first we've to separate a few highlights out of the picture.

Texture arrangement cares with recognizing a given finished area from a given arrangement of surface classes. Every one of those locales has exceptional surface attributes. There are three primary methodologies in Texture order:

(i)Structural

(ii)Statistical

(iii)Modelling approach

Statisticalsurface depiction techniques characterize the vibe upheld portraying the spatial dissemination of dark qualities processing neighborhood highlights at each point inside the picture, and inferring a gathering of insights from the dispersions of the nearby highlights. Nearby highlights are characterized by the blend of powers at explicit position comparative with each point inside the picture. Insights are named an essential, second or higher request measurements predictable with the measure of focuses which characterize the neighborhood include. the main insights are the dim level first-request measurements. They depict the dark level histogram of an image. In first request insights, picture properties rely on singular pixel esteems. Second-request measurements like the co-event framework strategy and accordingly the dim level contrast technique portray the spatial connections between picture pixels. In second request measurements, the picture properties rely on pixel sets. Higher request insights, including run length measures and in this way the autocorrelation work, likewise can be estimated for surface investigation. As opposed to auxiliary techniques, measurable methodologies don't plan to see unequivocally the progressive information structure of the surface.

Statisticaltechniques are broadly utilized. for example GLCM, contrast, entropy, homogeneity one among the methodology wont to characterize precisely what surface is factual surface. It might be a quantitative proportion of the plan of forces during an area. This arrangement of estimations is named a component vector.

GLCM: A gray level co-occurrence matrix (GLCM) contains data about the places of pixels having comparative dim level qualities. A co-event network might be a twodimensional cluster, $\mathrm{P}$, during which both the lines and in this manner the segments speak to a gathering of conceivable picture esteems. A GLCM $\operatorname{Pd}[i, j]$ is characterized by first determining a relocation vector $\mathrm{d}=(\mathrm{dx}, \mathrm{dy})$ and checking all sets of pixels isolated by $\mathrm{d}$ having dark levels I and j.

The GLCM is characterized by: $\operatorname{Pd}[\mathrm{i}, \mathrm{j}]=\mathrm{nij}$

where nij is that the quantity of events of the pixel esteems $(i, j)$ lying at separation $d$ inside the picture. The co-event lattice $\mathrm{Pd}$ has measurement $\mathrm{n} \times \mathrm{n}$, where $\mathrm{n}$ is that the quantity of dark levels inside the image.The calculation of the Coevent grid upheld picture pixels inside the structure framework as follows

\begin{tabular}{|l|l|l|l|}
\hline 0 & 0 & 1 & 1 \\
\hline 0 & 0 & 1 & 1 \\
\hline 0 & 2 & 2 & 2 \\
\hline 2 & 2 & 3 & 3 \\
\hline 2
\end{tabular}$\longrightarrow$\begin{tabular}{|l|l|l|l|l|l|}
\hline ij & 0 & 1 & 2 & 3 \\
\hline 0 & $\#(0,0)$ & $\#(0,1)$ & $\#(0,2)$ & $\#(0,3)$ \\
\hline 1 & $\#(1,0)$ & $\#(1,1)$ & $\#(1,2)$ & $\#(1,3)$ \\
\hline 2 & $\#(2,0)$ & $\#(2,1)$ & $\#(2,2)$ & $\#(2,3)$ \\
\hline 3 & $\#(3,0)$ & $\#(3,1)$ & $\#(3,2)$ & $\#(3,3)$ \\
\hline
\end{tabular}

Pixel values $=0,1,2,3$

so $\mathrm{N}=4$

size of co-occurrence matrix $=4 * 4$

Figure 3:Finding the no of occurrences of pixel $i$ to the neighbouring pixel value $\mathrm{j}$

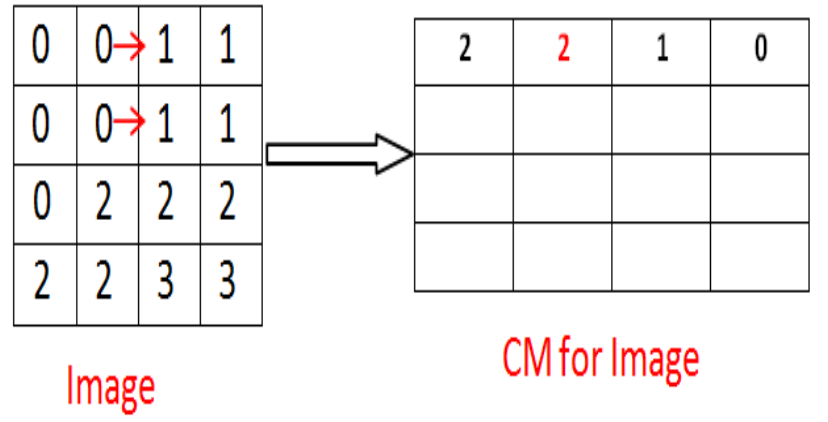

Figure 4:Computation of Co-occurrence Matrix

\section{Algorithm For GLCM:}

Step 1: A picture consists of pixels each with an intensity at a selected gray level and Quantize the input image into number of intensity of gray levels

Step2:Find a co-occurrence matrix for given image .Coevent network ought to be twodimensional cluster (i.e) lattice $(\mathrm{NxN})$

Step 3:Count all sets of pixels during which the essential pixel includes a worth I, and its coordinating pair dislodged from the essential pixel by $\mathrm{d}$ includes an estimation of $\mathrm{j}$. This tally is entered inside the ith line and jth segment of the framework $\mathrm{G} \mathrm{d}[\mathrm{i}, \mathrm{j}]$

Stage 4: Make the GLCM symmetric, Note that Gd[i,j] isn't symmetric, since the measure of sets of pixels having dark levels $[i, j]$ doesn't really rise to the measure of pixel sets having grey levels $[\mathrm{j}, \mathrm{i}]$. 
Step 5:Normalize the GLCM :Each component is part by entirety, everything being equal, $\mathrm{N}[\mathrm{i}, \mathrm{j}]=\frac{\mathrm{G}[\mathrm{i}, \mathrm{j}] \mathrm{f}}{\sum \mathrm{i} \sum \mathrm{j} \mathrm{j}[\mathrm{i}, \mathrm{j}]}$

\subsection{CNN Classifier:}

\subsubsection{Convolutional Layer}

The convolution activity is that the first of numerous changes performed during a convolutional layer of CNNs. For the most part, the convolution activity are frequently portrayed as computing the aggregate of items between a gathering of info esteems and estimations of a convolution piece, additionally called a channel.

In CNN, the bit qualities are prepared to search out ideal highlights from the motivation behind perspective on the assignment to be tackled (for our situation, foreseeing crop yield). To apply this channel to an image , an info picture, $\mathrm{F}(\mathrm{x}, \mathrm{y})$, is convolved with the piece, $\mathrm{K}$. Convolution is spoken to by a bullet (not to be confused with increase). It includes taking a bit, which is our little network of numbers, and spending it over an image, pixel-by-pixel, making another edge-identified yield picture whose appearance relies upon the portion esteems.

The means for a whole convolution are as per the following:

1.Multiply the qualities inside the portion with their coordinating pixel esteem. Thus, the value inside the upper left of the $3 \times 3$ portion (0), will be increased by the pixel esteem in that equivalent corner in our picture zone (150).

2.Sum of these duplicated sets of qualities to encourage a substitution esteem, during this case, 175 . This worth will be the new pixel esteem inside the separated yield picture, at a comparable $(x, y)$ area in light of the fact that the chose focus pixel.

This cycle rehashes for every pixel inside the info picture, until we are left with a whole, sifted yield.

The info picture is diminished to a littler size utilizing a channel. The channel is moved bit by bit running from the upper left corner of the picture. At each progression, the qualities inside the picture are duplicated by the estimations of the channel and consequently the outcome's added. a supplanting framework with a littler size is produced using the information picture. Fig. 5 shows the convolution activity inside the convolution layer for a $5 \times 5$ info picture and a $3 \times 3$ channel

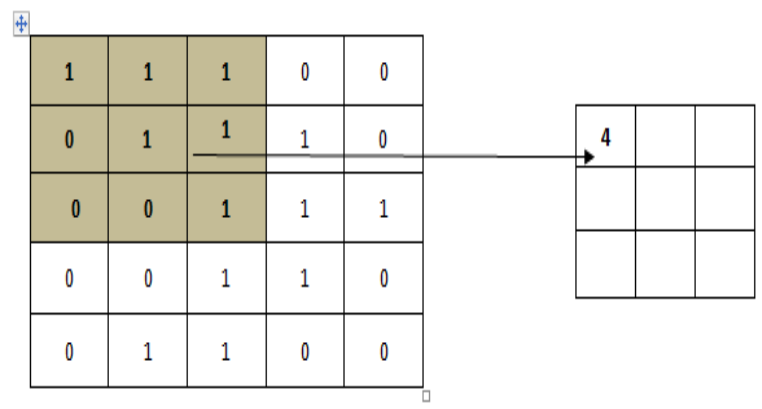

\begin{tabular}{|c|c|c|c|c|c|c|}
\hline 1 & 1 & 1 & 0 & 0 & & \\
\hline 0 & 1 & 1 & 1 & 0 & 4 & 3 \\
\hline 0 & 0 & 1 & 1 & 1 & & \\
\hline 0 & 0 & 1 & 1 & 0 & & \\
\hline 0 & 1 & 1 & 0 & 0 & & \\
\hline
\end{tabular}

Fig.5. Convolution operation for 5X5 input image and $3 \times 3$ filter

\subsubsection{Pooling Layer}

The use of pooling activity assists with separating a blend of highlights, which are invariant to translational movements and little. The pooling layer is normally applied after the convolution layer. the components of the yield network acquired from the convolution layer is decreased during this layer. Despite the fact that channels of different sizes are frequently used in the pooling layer, by and large $2 \times 2$ size channel is utilized. A few capacities like max pooling, normal pooling and L2-standard pooling are frequently used in this layer. during this examination, max pooling channel with step 2 has been applied. Max pooling is finished by choosing the most significant incentive inside the sub windows and this worth is moved to during another grid. Fig. 6 shows a model pooling activity.

\begin{tabular}{|l|l|l|l|}
\hline 2 & 3 & 1 & 5 \\
\hline 5 & 6 & 7 & 8 \\
\hline 3 & 5 & 1 & 1 \\
\hline 2 & 4 & 3 & 6 \\
\hline
\end{tabular}$\rightarrow$\begin{tabular}{|l|l|}
\hline 6 & 8 \\
5 & 6 \\
\hline
\end{tabular}

Fig.6. Max pooling with 2X2 filters and stride

\subsubsection{Fully Connected Layer}

The last obtained matrix, after finishing the convolution, pooling and activation operations, is fed into the fully connected layer as input. Recognition and classification are 
performed during this layer

\section{Result and Discussion:}

To begin making forecasts with highlights to be specific texture,color,shape of the leaves by applying testing dataset inside the model you will utilize a disarray network to see the measure of right and mistaken expectations. A disarray grid, additionally alluded to as a slip-up lattice, might be a network that reports the measure of genuine positives(tp), bogus positives(fp), genuine negatives(tn), and bogus negatives(fn) of a classifier.

\begin{tabular}{|l|l|l|}
\hline & Healthy Predicted & Disease Predicted \\
\hline Class 1- Healthy & 758 & 242 \\
1000 & & \\
\hline Class 2 -Diseased & 96 & 824 \\
\hline
\end{tabular}

The accuracy of the CNN model is predicted by $82.39 \%$

$$
\text { Accuracy }=\frac{\mathrm{TP}+\mathrm{TN}}{\mathrm{TP}+\mathrm{TN}+\mathrm{FP}+\mathrm{FN}}
$$

\section{Class 1- Healthy (1000)}

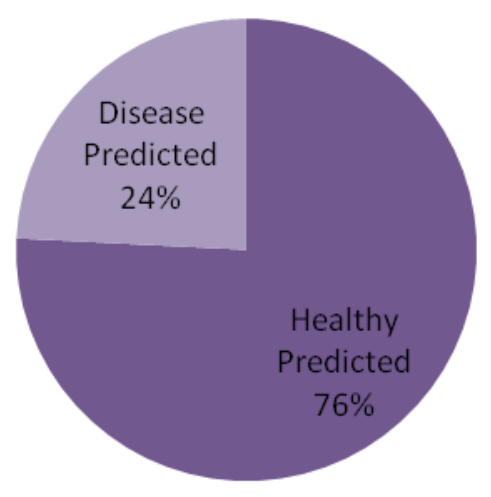

Fig.7.Accuracy prediction of TP and TN

\section{Class 2 -Diseased (920)}

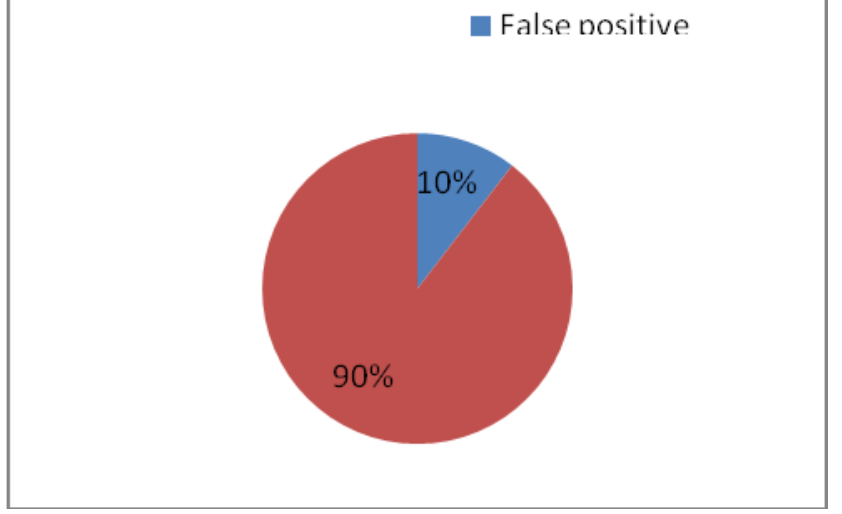

Fig.8.Accuracy prediction of FP and FN

- A genuine positive is a result where the model accurately predicts the positive class (likewise alluded to as affectability or review).

- A genuine negative is a result where the model effectively predicts the negative class.

- A bogus positive is a result where the model inaccurately predicts the positive class.

- A bogus negative is a result where the model inaccurately predicts the negative class.

The disarray framework yield implies your profound learning model made $758+824$ right forecasts and $242+96$ wrong expectations. you'll figure the exactness with: (758+ $824) / 1920$. the whole number of perceptions in your dataset is 1920. this gives you a precision of 82.39. Fig.7. what's more, Fig.8. shows proposed model accomplishing at least $82 \%$ right expectations from the given dataset.

\section{Conclusion}

Plant Diseases are major food threats that ought to need to overcome before it results in further loss of the whole field. But, often framers unable to differentiate between similar symptoms but ace different diseases. With the assistance of the model, we are ready to predict the crop disease at the sooner stage, thereby aids in increased crop yield and avoid the over dosage of fertilizers. within the future work, we'll attempt to apply activation functions on different crops to enhance the model further.

\section{References}

[1] J. Amara et al, "A deep learning-based approach for banana leaf diseases classification," in Proceedings of the Datenbanksysteme für Business, Technologie und Web (BTW '17) Workshopband, 2017.

[2] Ferentinos et al, "Deep learning models for plant disease detection and diagnosis," Computers and 
Electronics in Agriculture, vol. 145, pp. 311-318, 2018. View at Publisher - View at Scopus · View at Google Scholar

[3] Sladojevic et al, "Deep neural networks based recognition of plant diseases by leaf image classification," Computational Intelligence and Neuroscience, vol. 2016, Article ID 3289801, 11 pages, 2016. View at Publisher. View at Scopus · View at Google Scholar

[4] G. Wang et al, "Automatic image-based plant disease severity estimation using deep learning," Computational Intelligence and Neuroscience, vol. 2017, Article ID 2917536, 8 pages, 2017. View at Publisher - View at Google Scholar

[5] A. Ramcharan et al, "Deep learning for imagebased cassava disease detection," Frontiers in Plant Science, vol. 8, p. 1852, 2017. View at Scopus · View at Google Scholar

[6] A. Fuentes et al, “A robust deep-learning-based detector for real-time tomato plant diseases and pests recognition," Sensors, vol. 17, no. 9, p. 2022, 2017. View at Publisher - View at Google Scholar

[7] E. Fujita et al, "Basic investigation on a robust and practical plant diagnostic system," in Proceedings of 2016 15th IEEE International Conference on Machine Learning and Applications (ICMLA), pp. 989-992, 2016. View at Scopus

[8] Shima Ramesh et al "Plant Disease Detection Using Machine Learning " 2018 International Conference on Design Innovations for 3Cs Compute Communicate Control978-1-5386-75236/18/\$31.00 @2018 IEEE

[9] R. Sathish, R. Manikandan, S. Silvia Priscila, B. V. Sara and R. Mahaveerakannan, "A Report on the Impact of Information Technology and Social Media on Covid-19," 2020 3rd International Conference on Intelligent Sustainable Systems (ICISS), Thoothukudi, India, 2020, pp. 224-230, doi: 10.1109/ICISS49785.2020.9316046.

[10]Xudong et al (2017) The survey of object detection based on convolutional neural networks. Appl Res Comput 34(10): 2881-2886 + 2891 .

[11]Manikandan, $\mathrm{R}$ and Dr.R.Latha (2017). “A literature survey of existing map matching algorithm for navigation technology. International journal of engineering sciences \& research technology", 6(9), 326-331.Retrieved September $15,2017$.
[12]Aamir et al (2018) A hybrid proposed framework for object detection and classification. J Inf Process Syst 14(5):1176-1194 